\title{
Cutaneous Pili Migrans
}

\author{
Yoon Hwan Kim, Jung In Kim, Sang Hyeon Hwang, Joon Hong Min, Seoung Wan Chae', \\ Seung Jae Lee, Ga-Young Lee, Won-Serk Kim, Ji-Hye Park \\ Departments of Dermatology and ${ }^{1}$ Pathology, Kangbuk Samsung Hospital, Sungkyunkwan University School of Medicine, Seoul, Korea
}

\section{Dear Editor:}

Cutaneous pili migrans is a rare skin condition in which a hair shaft or fragment is embedded in the superficial skin. It is characterized by a creeping eruption with black-line like hair mimicking cutaneous larva migrans ${ }^{1}$. Removal of the black hair shaft heals the lesion completely ${ }^{2,3}$. Herein, we report a case of cutaneous pili migrans with the clinical and histopathological findings.

A 46-year-old Korean man presented with a 3-week his- tory of asymptomatic linear eruption along the crease of the neck. A thin black, thread-like line resembling a hair shaft was embedded below the epidermis. He had no suspicious history such as trauma. A punch biopsy was taken from the edge of the eruption, and a small incision was made at the other end. The black line was easily extracted, and it was found to be a hair shaft (Fig. 1). Histopathological examination revealed a cross-sectioned hair fragment in a tiny empty space in the superficial dermis
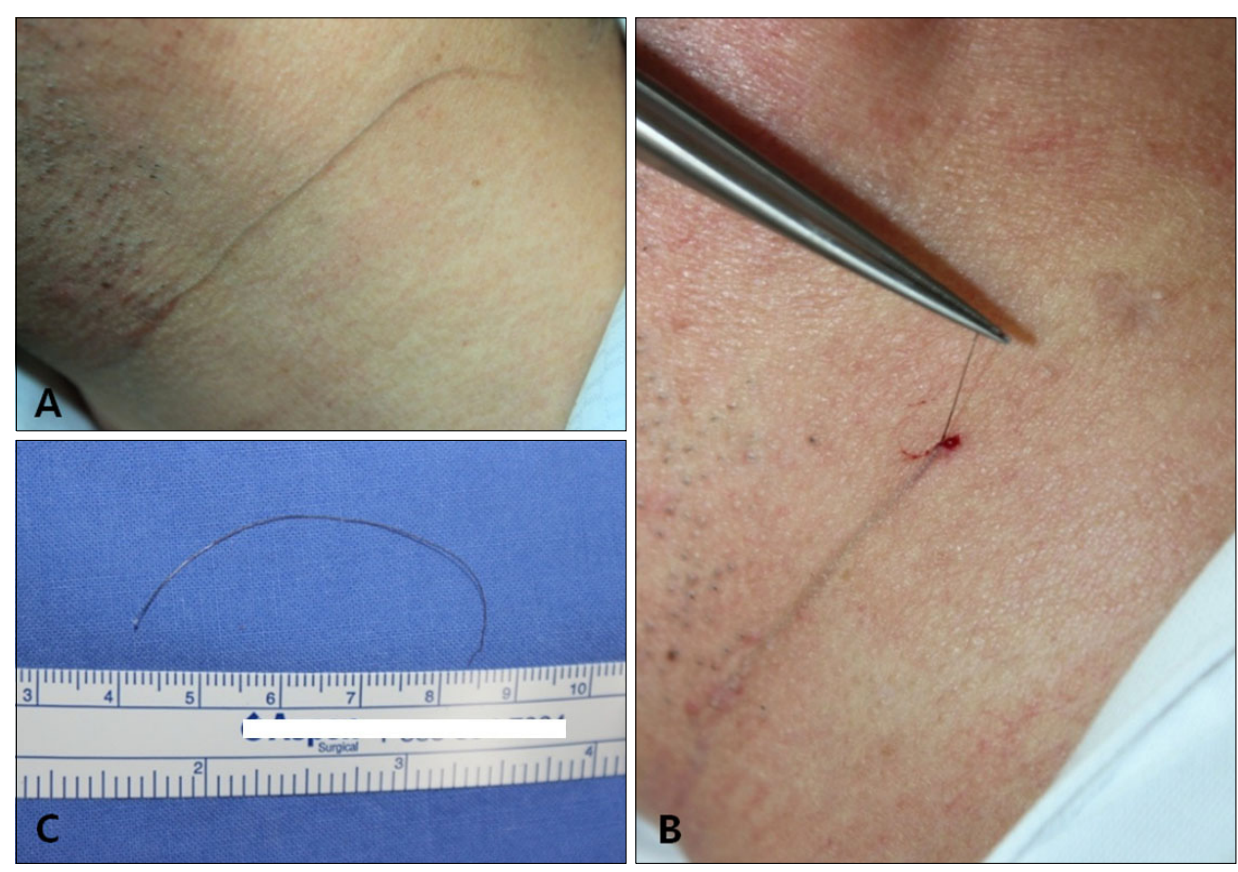

Fig. 1. (A) A slightly raised, linear, black eruption on the neck. (B) The black line was easily removed by making a shallow incision on the skin, and it was demonstrated to be a hair shaft. (C) The extracted hair shaft, measuring approximately 7 $\mathrm{cm}$.

Received April 25, 2013, Revised July 25, 2013, Accepted for publication August 3, 2013

Corresponding author: Ji-Hye Park, Department of Dermatology, Kangbuk Samsung Hospital, Sungkyunkwan University School of Medicine, 29 Saemunan-ro, Jongno-gu, Seoul 110-746, Korea. Tel: 82-2-2001-2228, Fax: 82-2-2001-2236, E-mail: wisdom0920@naver.com

This is an Open Access article distributed under the terms of the Creative Commons Attribution Non-Commercial License (http:// creativecommons.org/licenses/by-nc/3.0) which permits unrestricted non-commercial use, distribution, and reproduction in any medium, provided the original work is properly cited. 


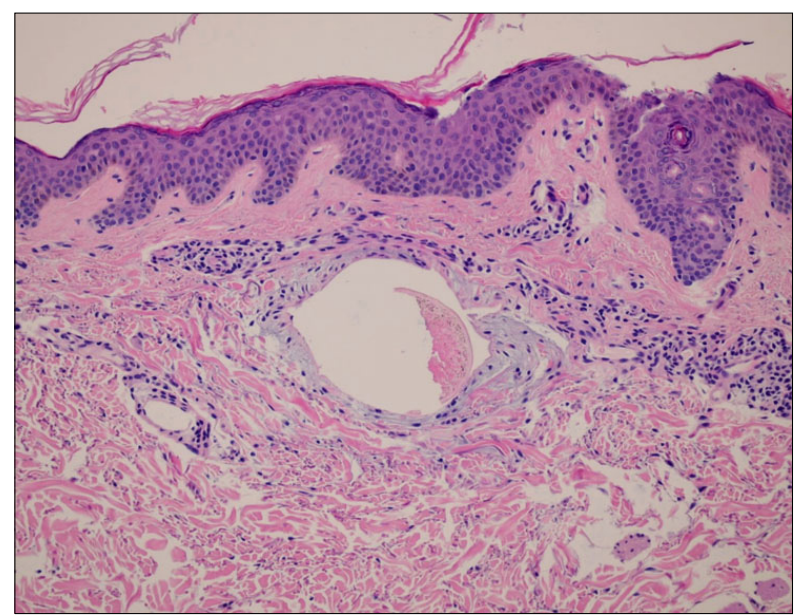

Fig. 2. Histopathologic finding shows a cross-sectioned hair fragment in a tiny empty space in the superficial dermis $(\mathrm{H} \& \mathrm{E}$, $\times 100)$.

(Fig. 2). A diagnosis of cutaneous pili migrans was made. After the burrowing hair shaft was removed, the eruption diminished immediately. At 4 months after the procedure, the patient recovered without any signs of recurrence.

Since 1957, 26 cases of cutaneous pili migrans have been described by using various terms such as burrowing hair, creeping hair, migrating hair, embedded hair, and bristle migrans ${ }^{1-4}$. Various locations have been reported, including the ankle, sole, toe, breast, cheek, neck, jaw, and abdomen. Although the etiology of the condition remains unclear, it is possibly an acquired condition because no hair follicles have been reported in all the reported cases. In the present case, only a hair shaft or a fragment was noted without any hair follicles. This is thought to be attributed to the hair shaft penetrating the epidermis owing to friction ${ }^{3}$. Franbourg et al..$^{5}$ reported that Asian hair has high tensile strength and the largest cross-sectional area compared with that of all other ethnic groups. This may explain why most of the cases were reported from East Asian countries. It is important to distinguish between cutaneous pili migrans and cutaneous larva migrans because the two conditions closely resemble each other. Cutaneous pili migrans tends to move in a linear fashion in only one direction. Cutaneous larva migrans can move in any direction and generally results in more serpinginous or tortuous tracts. Furthermore, lesions in cutaneous larva migrans are extremely itchy, whereas those in cutaneous pili migrans may be either asymptomatic or painful ${ }^{2}$. Therefore, it is necessary to recognize cutaneous pili migrans from other creeping eruptions via close examination.

\section{REFERENCES}

1. Yaffee HS. Imbedded hair resembling larva migrans. AMA Arch Derm 1957;76:254.

2. Sakai R, Higashi K, Ohta M, Sugimoto $Y$, Ikoma $Y$, Horiguchi $Y$. Creeping hair: an isolated hair burrowing in the uppermost dermis resembling larva migrans. Dermatology 2006;213:242-244.

3. Ishida Y, Matsubara K, Takai M, Horiguchi Y, Yoshikawa Y. A case of 'creeping hair' resembling cutaneous larva migrans. Clin Exp Dermatol 2009;34:256-257.

4. Luo DQ, Liu JH, Huang YB, He DY, Zhang HY. Cutaneous pili migrans: a case report and review of the literature. Int J Dermatol 2009;48:947-950.

5. Franbourg A, Hallegot P, Baltenneck F, Toutain C, Leroy F. Current research on ethnic hair. J Am Acad Dermatol 2003;48(6 Suppl):S115-S119. 